\title{
Ordered Ramsey theory and track representations of graphs
}

\author{
Kevin G. Milans, Derrick Stolee, and Douglas B. West*
}

\begin{abstract}
We study an ordered version of hypergraph Ramsey numbers for linearly ordered vertex sets, due to Fox, Pach, Sudakov, and Suk. In the $k$-uniform ordered path, the edges are the sets of $k$ consecutive vertices in a linear order. Moshkovitz and Shapira described its ordered Ramsey number in terms of an enumerative problem: it equals 1 plus the number of elements in the poset obtained by starting with a certain disjoint union of chains and repeatedly taking the poset of down-sets, $k-1$ times. After presenting a proof of this and the resulting bounds, we apply the bounds to study the minimum number of interval graphs whose union is the line graph of the $n$-vertex complete graph, proving the conjecture of Heldt, Knauer, and Ueckerdt that this grows with $n$. In fact, the growth rate is between $\Omega\left(\frac{\log \log n}{\log \log \log n}\right)$ and $O(\log \log n)$.
\end{abstract}

AMS 2000 SUBJECT ClASsifications: Primary 05C55, 05C62; secondary $05 \mathrm{C} 65,05 \mathrm{C} 35$.

\section{Introduction}

We study an ordered version of Ramsey numbers for hypergraphs. An ordered hypergraph is a hypergraph on a linearly ordered vertex set. An ordered hypergraph $H$ occurs as a subhypergraph of an ordered hypergraph $H^{\prime}$ if some order-preserving injection from $V(H)$ to $V\left(H^{\prime}\right)$ also preserves edges. We then say that $H^{\prime}$ contains (a copy of) $H$. Two ordered hypergraphs are isomorphic if each is a subhypergraph of the other.

Ramsey's Theorem concerns edge-colorings of ordinary (unordered) hypergraphs. Let $K_{r}^{k}$ denote the complete $k$-uniform hypergraph with $r$ vertices. For $t, k, r \in \mathbb{N}$, Ramsey's Theorem [19] implies that when $n$ is sufficiently large, every $t$-coloring of $E\left(K_{n}^{k}\right)$ contains a monochromatic copy of $K_{r}^{k}$ (and hence also of every subhypergraph of $K_{r}^{k}$ ). Because any complete ordered hypergraphs with the same number of vertices are isomorphic, the same statement holds in the ordered sense, including the part about subhypergraphs. This permits an ordered version of Ramsey numbers.

\footnotetext{
${ }^{*}$ Research supported by Recruitment Program of Foreign Experts, 1000 Talent Plan, State Administration of Foreign Experts Affairs, China.
} 
Definition 1.1. Let $H_{1}, \ldots, H_{t}$ be ordered $k$-uniform hypergraphs, where $k \geq 1$. The $t$-color ordered Ramsey number $\mathrm{OR}\left(H_{1}, \ldots, H_{t}\right)$ is the least integer $n$ such that in every $t$-coloring of the edges of the ordered hypergraph $K_{n}^{k}$ there is a copy of $H_{i}$ in color $i$ for some $i$. When $H_{1}=\cdots=H_{t}=H$, we abbreviate the notation to $\mathrm{OR}_{t}(H)$; this is the diagonal case. For a $k$ uniform ordered hypergraph $H$, an edge-coloring of the ordered hypergraph $K_{n}^{k}$ is $H$-avoiding if it has no monochromatic $H$. The $k$-uniform ordered path $P_{r}^{k}$ is the ordered hypergraph with $r$ vertices in which the edges are the intervals of $k$ consecutive vertices in the vertex ordering. Let $m$ be the number of edges in $P_{r}^{k}$, so $m=r-k+1$.

Note that $\mathrm{OR}_{t}\left(K_{p}^{k}\right)$ equals the usual $t$-color (unordered) Ramsey number for $K_{p}^{k}$. Although $\mathrm{OR}\left(H_{1}, \ldots, H_{t}\right)$ is defined for general ordered $k$-uniform hypergraphs $H_{1}, \ldots, H_{t}$, the problem is particularly fundamental for ordered paths, because this case has applications (see [9]) and reduces to computing the size of a natural poset. (Choudum and Ponnusamy [3] used "ordered Ramsey number" for a different concept about colored tournaments.)

Focusing on ordered paths, Fox, Pach, Sudakov, and Suk [9] defined $N_{k}(t, m)$ to be the least integer $N$ such that in every $t$-coloring of the $k$ subsets of $N$ linearly ordered elements there is a monochromatic $k$-uniform ordered path with $m$ edges. That is, $N_{k}(t, m)=\mathrm{OR}_{t}\left(P_{m+k-1}^{k}\right)$ in our notation. They proved $2^{(m / t)^{t-1}} \leq N_{3}(t, m) \leq 2^{m^{t-1} \lg m}$.

Eliáš and Matoušek [6] asked more generally for bounds on $N_{k}(t, m)$. Moshkovitz and Shapira [18] gave upper and lower bounds that are towers of the same height. For a nonnegative integer $h$, define $\operatorname{tow}_{h}(x)$ by

$$
\operatorname{tow}_{h}(x)=\left\{\begin{array}{ll}
2^{\operatorname{tow}_{h-1}(x)} & h \geq 1 \\
x & h=0
\end{array} .\right.
$$

The subscript in our tow tow $_{h}(x)$ is the number of $2 \mathrm{~s}$ in the tower; thus tow $1(x)$ is exponential in $x$ and tow $2(x)$ is "doubly-exponential" in $x$. In our notation, their bounds are as follows:

Theorem 1.2 (Moshkovitz and Shapira [18]). For $t \geq 2, k \geq 3$, and $m=$ $r-k+1 \geq 2$,

$$
\operatorname{tow}_{k-2}\left(m^{t-1} / 2 \sqrt{t}\right) \leq \operatorname{OR}_{t}\left(P_{r}^{k}\right) \leq \operatorname{tow}_{k-2}\left(2 m^{t-1}\right) .
$$

For $k=3$ they gave the sharper bounds $2^{\frac{2}{3} m^{t-1} / \sqrt{t}} \leq \mathrm{OR}_{t}\left(P_{r}^{3}\right) \leq 2^{2 m^{t-1}}$.

Their bounds arose by characterizing $\operatorname{OR}_{t}\left(P_{r}^{k}\right)$ using an enumerative problem. They converted this problem to a statement about down-sets in 
posets, which is where we start. They stated the result only for the diagonal case of paths with equal size, but they commented that their argument extends to the general case. Indeed, the general case is no more difficult. Thus it is proper to view it as due to Moshkovitz and Shapira [18]. Indeed, the proof we give here uses essentially the same ideas as their proof. We provide a concise alternative presentation for completeness in order to apply the resulting bounds, using notation that permits cleaner statements.

In order to state the result, we need terminology from posets. We view a partially ordered set or poset as a set equipped with an order relation. Let $Q$ be a poset. A chain in $Q$ is a set of pairwise comparable elements, and an antichain is a set of pairwise incomparable elements. A down-set in $Q$ is a set $S \subseteq Q$ such that if $y \in S$ and $x \leq y$, then $x \in S$. Let $|Q|$ denote the number of elements in $Q$. It is common to use $J(Q)$ to denote the containment poset on the set of down-sets of $Q$. Let $J^{k}(Q)$ denote the poset obtained from $Q$ by repeatedly taking the poset of down-sets, $k$ times. That is, $J^{0}(Q)=Q$ and $J^{k}(Q)=J\left(J^{k-1}(Q)\right)$ for $k \geq 1$.

Theorem 1.3. Given $k \in \mathbb{N}$, let $r_{1}, \ldots, r_{t}$ be positive integers greater than k. Let $m_{i}=r_{i}-k+1$ (thus $m_{i}=\left|E\left(P_{r_{i}}^{k}\right)\right|$ ) and let $Q$ be the poset consisting of disjoint chains with sizes $m_{1}-1, \ldots, m_{t}-1$. The ordered Ramsey number satisfies $\operatorname{OR}_{t}\left(P_{r_{1}}^{k}, \ldots, P_{r_{t}}^{k}\right)=\left|J^{k-1}(Q)\right|+1$.

A down-set in this poset $Q$ is formed by taking some nonnegative number of elements from the bottom of each chain in $Q$; hence $J(Q)$ is the product of chains of sizes $m_{1}, \ldots, m_{t}$. When $t=2$, a down-set in $J(Q)$ can be viewed as an integer partition. For general $t$ and $k$, Moshkovitz and Shapira discussed the problem using higher-dimensional "line partitions", where line partitions are higher-order analogues of integer partitions. They employed bijections to lattices of down-sets of appropriate posets. The result is easier to state and prove directly in terms of down-sets as given above. The inductive nature of $J^{k-1}(Q)$ yields recurrences for upper and lower bounds on $\mathrm{OR}_{t}\left(P_{r}^{k}\right)$ as immediate corollaries, which in turn yield the bounds in [18].

The resulting lower bound on $\mathrm{OR}_{t}\left(P_{r}^{k}\right)$ applies to the problem that motivated our study. In Section 3, we prove a conjecture of Heldt, Knauer, and Ueckerdt [13] about geometric representations of graphs. A t-interval representation of a graph $G$ assigns each vertex the union of at most $t$ intervals on the real line so that vertices are adjacent if and only if their assigned sets intersect. The interval number $i(G)$ is the least $t$ such that $G$ has a $t$-interval representation. When $i(G)=1$, we say that $G$ is an interval graph and that the assignment of intervals is an interval representation. 
A $t$-track representation of $G$ is an expression of $G$ as the union of $t$ interval graphs. The track number $\tau(G)$ (apparently introduced in [12]) is the least $t$ such that $G$ has a $t$-track representation. Note that a $t$-track representation yields a $t$-interval representation by placing interval representations of the $t$ tracks in disjoint segments of the real line; thus $i(G) \leq \tau(G)$. (Note that $\tau(G)$ is at most the number of caterpillars needed to decompose $G$; see also $[10,11,12,14,17]$.)

If $i(G)=1$, then also $\tau(G)=1$, since such graphs are interval graphs. On the other hand, $i\left(K_{5,3}\right)=2<\tau\left(K_{5,3}\right)$. Heldt, Knauer, and Ueckerdt [13] conjectured that the track number is not bounded by any function of the interval number, even when restricted to line graphs. Letting $L(G)$ denote the line graph of a graph $G$, always $i(L(G)) \leq 2$, since each edge of $G$ is incident to two vertices, and the edges incident to a common vertex can be given intervals having a common point. Since track representations are hereditary over induced subgraphs, the track number is unbounded for line graphs if and only if it is unbounded for $L\left(K_{n}\right)$.

To study $\tau\left(L\left(K_{n}\right)\right)$, we relate $t$-color ordered Ramsey numbers of particular 3-uniform ordered hypergraphs to $t$-track representations of $L\left(K_{n}\right)$. Letting $t$ be the track number of $L\left(K_{n}\right)$, we prove

$$
\mathrm{OR}_{t-3}\left(P_{4}^{3}\right) \leq n<\mathrm{OR}_{t}\left(P^{\prime}\right) \leq \mathrm{OR}_{t}\left(K_{6}^{3}\right)
$$

where $P^{\prime}$ is obtained from $P_{6}^{3}$ by adding the two edges $\{1,2,5\}$ and $\{2,5,6\}$. By inverting the relationship between $t$ and $n$, we obtain

$$
\Omega\left(\frac{\lg \lg n}{\lg \lg \lg n}\right) \leq \tau\left(L\left(K_{n}\right)\right) \leq O(\lg \lg n) .
$$

\section{Ordered Ramsey numbers for $k$-uniform paths}

Let $[r]=\{1, \ldots, r\}$. We will use $[r]$ as the vertex set of $P_{r}^{k}$, so the $r-k+1$ edges are the sets of $k$ consecutive elements of $[r]$. Note that $P_{r}^{2}$ is the ordinary $r$-vertex path with vertices indexed in order. To clarify the role of the vertex ordering, consider instead the 3 -vertex path with edges $\{1,2\}$ and $\{1,3\}$. Avoiding the target when $t$-coloring $E\left(K_{n}\right)$ requires the edges leaving vertex 1 to have distinct colors, so $n-1 \leq t$. Coloring edges by their higher endpoint achieves the bound, so the ordered Ramsey number equals $t+2$. For the monotone path $P_{3}^{2}$, Theorem 1.3 with $r_{1}=\cdots=r_{t}=3$ and $k=2$ yields the much higher value $2^{t}+1$.

For $k=2$ in general, Theorem 1.3 states $\mathrm{OR}_{t}\left(P_{m_{1}+1}^{2}, \ldots, P_{m_{t}+1}^{2}\right)=$ $1+\prod_{i=1}^{t} m_{i}$. This case is special enough that it also arose in the tournament 
model of Choudum and Ponnusamy [3] (stated without proof). It is a general form of the Erdös-Szekeres Theorem, as observed by Seidenberg [20] and by Moshkovitz and Shapira [18]. Its proof provides motivation for the general argument. To show the upper bound, let $E\left(K_{n}\right)$ be $t$-colored with no increasing copy of $P_{r_{i}+1}$ in color $i$, and compute for $x \in[n]$ the $t$-tuple of orders of longest paths reaching $x$ in the various colors; only $\prod_{i=1}^{t} r_{i}$ such $t$-tuples are available, and they must be distinct. To achieve equality, view $[n]$ as $\prod_{i=1}^{t}\left[r_{i}\right]$ and let the color of an edge be the first index where the endpoints differ.

In the proof of the general upper bound, we will need the smallest downset containing a given set of elements in a poset $Q$. For $S \subseteq Q$, the down-set generated by $S$, denoted $D(S)$, is $\{u \in Q: u \leq v$ for some $v \in S\}$. We restate Theorem 1.3 in a slightly different way to simplify notation in the proof.

Theorem 2.1. Given $k \in \mathbb{N}$, let $r_{1}, \ldots, r_{t}$ be positive integers greater than $k$. Let $Q$ be the poset consisting of disjoint chains with sizes $r_{1}-k, \ldots, r_{t}-k$. Letting $Q_{1}=Q$ and $Q_{i}=J\left(Q_{i-1}\right)$ for $i>1$, the ordered Ramsey number satisfies $\mathrm{OR}_{t}\left(P_{r_{1}}^{k}, \ldots, P_{r_{t}}^{k}\right)=\left|Q_{k}\right|+1$.

Proof. The case $k=1$ is just the pigeonhole principle; consider $k \geq 2$.

Lower bound. For $n=\left|Q_{k}\right|$, we will construct a $t$-edge-coloring of $K_{n}^{k}$ that avoids $P_{r_{i}}^{k}$ in color $i$ for each $i$. First consider $x, y \in Q_{j}$, where $2 \leq j \leq k$. If $x \nsupseteq y$, then $x$ does not contain $y$ when they are viewed as down-sets in $Q_{j-1}$; let $f(x, y)$ be a particular element of the set $y-x$ in $Q_{j-1}$. For $x, y, z \in Q_{j}$, if $x \nsupseteq y$ and $y \nsupseteq z$, then $y$ (in $Q_{j-1}$ ) contains $f(x, y)$ but not $f(y, z)$. Since $y$ is a down-set in $Q_{j-1}$, we obtain $f(x, y) \nsucceq f(y, z)$ in $Q_{j-1}$.

A list $x_{1}, \ldots, x_{s}$ in a poset is descent-free if $x_{i} \geq x_{i+1}$ for $1 \leq i \leq$ $s-1$. We extend $f$ to descent-free lists in $Q_{j}$ by setting $f\left(x_{1}, \ldots, x_{s}\right)=$ $\left(f\left(x_{1}, x_{2}\right), \ldots, f\left(x_{s-1}, x_{s}\right)\right)$. The image of a descent-free $s$-list in $Q_{j}$ is a descent-free $(s-1)$-list in $Q_{j-1}$. Let $f^{0}$ be the identity map. For a descentfree $s$-list $x_{1}, \ldots, x_{s}$ in $Q_{j}$, where $s, j>d>1$, define $f^{d}\left(x_{1}, \ldots, x_{s}\right)=$ $f\left(f^{d-1}\left(x_{1}, \ldots, x_{s}\right)\right)$. When defined, $f^{d}\left(x_{1}, \ldots, x_{s}\right)$ is a descent-free $(s-d)$ list in $Q_{j-d}$.

Now let $y_{1}, \ldots, y_{n}$ be a linear extension of $Q_{k}$; that is, an ordering of $Q_{k}$ such that $y_{i} \leq y_{j}$ implies $i \leq j$. Each sublist of a linear extension is descent-free. Also, for sublist $x_{1}, \ldots, x_{k}$, note that $f^{k-1}\left(x_{1}, \ldots, x_{k}\right)$ is a single element in $Q$. Color $\left\{x_{1}, \ldots, x_{k}\right\}$ (as an edge in $K_{n}^{k}$ ) with the index of the chain in $Q$ that contains $f^{k-1}\left(x_{1}, \ldots, x_{k}\right)$. If $x_{1}, \ldots, x_{s}$ is a sublist of $y_{1}, \ldots, y_{n}$ whose consecutive $k$-lists all have color $i$, then $f^{k-1}\left(x_{1}, \ldots, x_{s}\right)$ is a descent-free $(s-k+1)$-list in the $i$ th chain of $Q$. A descent-free list in a 
chain is strictly increasing, so $s-k+1 \leq r_{i}-k$. Thus $s<r_{i}$, and hence the coloring avoids $P_{r_{i}}^{k}$ in color $i$ for each $i$.

Upper bound. Given a $t$-edge-coloring $\phi$ of $E\left(K_{n}^{k}\right)$ with vertex set $[n]$ that avoids $P_{r_{i}}^{k}$ in color $i$ for each $i$, it suffices to define an injection from $[n]$ to $Q_{k}$. View each vertex subset $Y \subseteq[n]$ as an increasing list, with $Y^{-}=Y-\max Y$ and $Y^{+}=Y-\min Y$.

For $1 \leq j \leq k<n$, we construct $g_{j}:\left(\begin{array}{c}{[n]} \\ j\end{array}\right) \rightarrow Q_{k-j+1}$ such that

$$
g_{j}\left(Y^{-}\right) \nsupseteq g_{j}\left(Y^{+}\right) \text {in } Q_{k-j+1} \text { when } Y \in\left(\begin{array}{c}
{[n]} \\
j+1
\end{array}\right) .
$$

This suffices, since $g_{1}$ will then be the desired injection.

We first define $g_{k}$. For $X \in\left(\begin{array}{c}{[n]} \\ k\end{array}\right)$, let $i=\phi(X)$, and let $w_{1}, \ldots, w_{r_{i}-k}$ be the $i$ th chain in $Q$. Set $g_{k}(X)=w_{h}$, where $h$ is the largest integer such that some $i$-colored copy of $P_{h+k-1}^{k}$ has last edge $X$. Note that $h \leq r_{i}-k$, since $\phi$ yields no $i$-colored copy of $P_{r_{i}}^{k}$. If $\phi\left(Y^{-}\right)=\phi\left(Y^{+}\right)$for some $Y \in\left(\begin{array}{c}{[n]} \\ k+1\end{array}\right)$, then $g_{k}\left(Y^{+}\right)>g_{k}\left(Y^{-}\right)$, and otherwise $g_{k}\left(Y^{+}\right)$and $g_{k}\left(Y^{-}\right)$are incomparable in $Q$. In either case, $g_{k}\left(Y^{-}\right) \nsupseteq g_{k}\left(Y^{+}\right)$.

Now consider smaller $j$, with $g_{j+1}$ already defined and satisfying $(*)$. For $X \in\left(\begin{array}{c}{[n]} \\ j\end{array}\right)$, let $g_{j}(X)=D\left(\left\{g_{j+1}(Y): Y^{+}=X\right\}\right)$. Since $Q_{k-j+1}=$ $J\left(Q_{k-j}\right)$, by construction $g_{j}(X) \in Q_{k-j+1}$. Now consider $Y \in\left(\begin{array}{l}{[n]} \\ j+1\end{array}\right)$; note that $g_{j+1}(Y) \in g_{j}\left(Y^{+}\right)$. If also $g_{j+1}(Y) \in g_{j}\left(Y^{-}\right)$, then since $g_{j}\left(Y^{-}\right)$is a down-set there exists $Z \in\left(\begin{array}{c}{[n]} \\ j+1\end{array}\right)$ such that $g_{j+1}(Y) \leq g_{j+1}(Z)$ in $Q_{k-j}$ and $Z^{+}=Y^{-}$. That is, $g_{j+1}\left(W^{-}\right) \geq g_{j+1}\left(W^{+}\right)$for $W=Y \cup Z$, which contradicts $(*)$ for $g_{j+1}$. Thus $g_{j+1}(Y) \in g_{j}\left(Y^{+}\right)-g_{j}\left(Y^{-}\right)$, which yields $g_{j}\left(Y^{-}\right) \nsupseteq g_{j}\left(Y^{+}\right)$.

With $Q$ being the disjoint union of chains of sizes $m_{1}-1, \ldots, m_{t}-1$, we have noted that $\left|Q_{1}\right|=\sum\left(m_{i}-1\right)$ and $\left|Q_{2}\right|=\prod m_{i}$, since $Q_{2}$ is the product of chains of sizes $m_{1}, \ldots, m_{t}$.

For $k \geq 3$, exact values are unknown. Since down-sets correspond bijectively to antichains, $\left|Q_{3}\right|$ is the number of antichains in the chain-product. As noted by Moshkovitz and Shapira, this is already a famous problem when all $m_{i}$ equal 2: counting the antichains among subsets of $[t]$ is known as Dedekind's Problem. The family of all subsets of $\left(\begin{array}{c}{[t]} \\ \lfloor t / 2\rfloor\end{array}\right)$ yields the lower bound $2^{\left(\begin{array}{c}t \\ t / 2\rfloor\end{array}\right)}$; the family of all subsets of $Q_{2}$ yields the upper bound $2^{2^{t}}$. Not surprisingly, the lower bound is sharper: again for the case where all $m_{i}$ equal 2, Kleitman and Markovsky [16] proved

$$
\left(\begin{array}{c}
t \\
\lfloor t / 2\rfloor
\end{array}\right) \leq \lg \left|Q_{3}\right| \leq\left(\begin{array}{c}
t \\
\lfloor t / 2\rfloor
\end{array}\right)\left(1+O\left(\frac{\log n}{n}\right)\right) .
$$


The upper bound was further refined by Korshunov [15]. Note that $\left(\begin{array}{c}t \\ \lfloor t / 2\rfloor\end{array}\right) \approx$ $2^{t} / \sqrt{2 \pi t}$ and $3>\sqrt{2 \pi}$ yield the lower bound on $\mathrm{OR}_{t}\left(P_{4}^{3}\right)$ given by Moshkovitz and Shapira.

Asymptotics are also known for the size of the largest rank in $Q_{2}$ when $m_{1}=\cdots=m_{t}=m>2$. As long as $m \in o\left(e^{t} / \sqrt{t}\right)$, the size is asymptotic to $m^{t-1} / \sqrt{\pi t / 6}$ (see [2]). Since $\sqrt{\pi / 6}<2$, for $t>\log m+\frac{1}{2} \log \log m$ this slightly improves the lower bound on $\mathrm{OR}_{t}\left(P_{m+2}^{3}\right)$ from Moshkovitz and Shapira [18] (stated in Theorem 1.2).

For larger $k$, we iterate the argument. Our point is that this is how the enumerative characterization yields the bounds of [18]. Using the constant 2 instead of $\sqrt{\pi / 6}$ allows in Theorem 1.2 a uniform lower bound for all $t$ without reference to the logarithmic term in the argument to the tower function.

Corollary 2.2. Let $Q$ be the disjoint union of thains of size $m-1$. Define $Q_{k}$ for $k \geq 1$ as in Theorem 2.1. For $k \geq 3$ and $t>\log m+\frac{1}{2} \log \log m$,

$$
\operatorname{tow}_{k-2}\left(m^{t-1} / \sqrt{\pi t / 6}-O\left(t^{2}\right)\right) \leq\left|Q_{k}\right| \leq \operatorname{tow}_{k-2}\left(m^{t}\right) .
$$

Proof. The upper bound arises from $\left|Q_{2}\right|=m^{t}$ by iteratively taking all subsets.

For the lower bound, we define an analogue of the tower function using the middle binomial coefficient. Let $b_{0}(x)=x$. For $h \geq 1$, let $b_{h}(x)=$ $\left(\begin{array}{c}b_{h-1}(x) \\ \left\lfloor b_{h-1}(x) / 2\right\rfloor\end{array}\right)$. In terms of the tower function, Stirling's approximation yields $b_{h}(x) \geq \operatorname{tow}_{h}(x-O(\lg x))$.

Let $a_{k}$ be the maximum size of a family in $Q_{k}$ consisting of down-sets in $Q_{k-1}$ that have the same size; these sets form an antichain in $Q_{k}$. Thus $\left|Q_{k+1}\right| \geq 2^{a_{k}}$, but also $a_{k+1} \geq\left(\begin{array}{c}a_{k} \\ \left\lfloor a_{k} / 2\right\rfloor\end{array}\right)$. Thus $a_{k} \geq b_{k-2}\left(a_{2}\right)$. With $a_{2}=$ $m^{t-1} / \sqrt{\pi t / 6}$, we have

$$
\left|Q_{k}\right| \geq 2^{a_{k-1}} \geq 2^{b_{k-3}\left(a_{2}\right)} \geq \operatorname{tow}_{k-2}\left(m^{t-1} / \sqrt{\pi t / 6}-O\left(t^{2}\right)\right) .
$$

Since we do not have an exact computation of $\left|J^{k-1}(Q)\right|$, recursive bounds may be of interest. In an earlier version of this paper, methods like those of Theorem 2.1 were used to give short proofs of several such bounds.

Theorem 2.3. For $m=r-k+1$ and $r>k \geq 3$, the following recursive bounds hold:

$$
\begin{aligned}
& \mathrm{OR}_{t}\left(P_{r+1}^{k}\right) \geq \mathrm{OR}_{\left(\begin{array}{c}
t \\
\lfloor t / 2\rfloor
\end{array}\right)}\left(P_{r}^{k-1}\right) \quad \mathrm{OR}_{t}\left(P_{k+1}^{k}\right) \leq \mathrm{OR}_{2^{t}}\left(P_{k}^{k-1}\right) \\
& \mathrm{OR}_{t}\left(P_{r}^{k}\right) \leq \mathrm{OR}_{m^{t}}\left(P_{k}^{k-1}\right) \quad \mathrm{OR}_{t}\left(P_{k+2}^{k}\right) \leq \operatorname{OR}_{2^{2 t}}\left(P_{k}^{k-1}\right) .
\end{aligned}
$$


It would be interesting to obtain good bounds on other ordered Ramsey numbers. For example, an ordered $k$-uniform version of a cycle can be defined by letting the edges of $C_{r}^{k}$ with vertex set $[r]$ be all sets of $k$ members of $[r]$ whose congruence classes modulo $r$ are consecutive. Is $\mathrm{OR}_{t}\left(C_{r}^{k}\right)$ much larger than $\mathrm{OR}_{t}\left(P_{r}^{k}\right)$ ? Also, the ordinary Ramsey number of a graph is bounded by the ordered Ramsey numbers of all its orderings. Given an unordered graph, which vertex orderings lead to the smallest and largest ordered Ramsey numbers? For steps in these directions, see Balko and Král [1]. Another paper studying special cases of ordered Ramsey numbers for graphs (2-uniform hypergraphs) is [4].

\section{Separating interval number and track number}

Recall from the introduction the definitions of $t$-interval representation, $t$ track representation, interval number $i(G)$, and track number $\tau(G)$ for a graph $G$. We observed that always $i(G) \leq \tau(G)$. Heldt, Knauer, and Ueckerdt [13] conjectured that $\tau(G)$ can grow while $i(G)=2$ even for the line graphs of complete graphs. We prove this in the next theorem, using ordered Ramsey numbers. First we pose a stronger conjecture:

Conjecture 3.1. For a sequence $\left(G_{n}\right)_{n=1}^{\infty}$ of graphs, if $\chi\left(G_{n}\right) \rightarrow \infty$, then $\tau\left(L\left(G_{n}\right)\right) \rightarrow \infty$.

Theorem 3.2. Let $P^{\prime}$ be the ordered hypergraph obtained from the ordered $P_{6}^{3}$ with vertices $\{1, \ldots, 6\}$ by adding the edges $\{1,2,5\}$ and $\{2,5,6\}$. If $n \geq$ $\mathrm{OR}_{t}\left(P^{\prime}\right)$, then $\tau\left(L\left(K_{n}\right)\right)>t$.

Proof. From a $t$-track representation of $L\left(K_{n}\right)$, we define a $t$-coloring $\phi$ of $E\left(K_{n}^{3}\right)$. For $x, y, z \in[n]$ with $x<y<z$, let $\phi(x, y, z)$ be the index of a track where the intervals for $x y$ and $y z$ intersect. Since $x y$ and $y z$ are incident in $K_{n}, \phi$ is well-defined. The intervals for disjoint pairs do not overlap in any track, since they are not adjacent in $L\left(K_{n}\right)$.

Since $n \geq \operatorname{OR}_{t}\left(P^{\prime}\right)$, under $\phi$ there is a monochromatic ordered copy of $P^{\prime}$; let its vertices be $\left\{x_{1}, \ldots, x_{6}\right\}$ in order, and let its edges have color $s$. The intervals for $x_{i} x_{i+1}$ and $x_{j} x_{j+1}$ in track $s$ overlap when $|i-j|=$ 1 , but not when $|i-j|>1$. Hence we have a path of five successively overlapping intervals. Since this copy of $P^{\prime}$ is monochromatic, the interval for $x_{2} x_{5}$ must intersect the intervals for $x_{1} x_{2}$ and $x_{5} x_{6}$, but it must not intersect the interval for $x_{3} x_{4}$ that lies between them. Hence these incidences cannot be represented in a single track, and $L\left(K_{n}\right)$ has no $t$-track representation. 
Among classical Ramsey numbers, Erdős and Rado [8] proved $R_{t}\left(K_{6}^{3}\right) \leq$ $2^{2^{O(t \lg t)}}$. Conlon, Fox, and Sudakov [5] reduced the constant, proving $R_{t}\left(K_{6}^{3}\right) \leq 2^{2^{(4+o(1)) t \lg t}}$.

Corollary 3.3. $\tau\left(L\left(K_{n}\right)\right) \geq \frac{\lg \lg n}{(4+o(1)) \lg \lg \lg n}$.

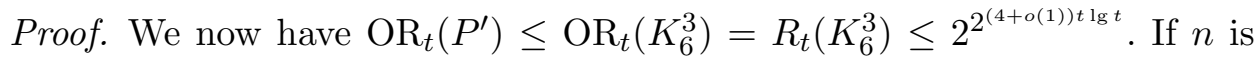
larger than this bound, then $n>\mathrm{OR}_{t}\left(P^{\prime}\right)$ and $\tau\left(L\left(K_{n}\right)\right)>t$. To express

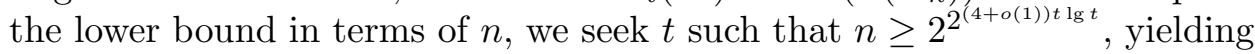
the claimed bound.

For an upper bound on $\tau\left(L\left(K_{n}\right)\right)$, we again use ordered Ramsey theory.

Theorem 3.4. If $n<\mathrm{OR}_{t}\left(P_{4}^{3}\right)$, then $\tau\left(L\left(K_{n}\right)\right) \leq t+2$.

Proof. We convert a $P_{4}^{3}$-avoiding $t$-coloring $\phi$ of $E\left(K_{n}^{3}\right)$ into a representation of $L\left(K_{n}\right)$ with $t+2$ tracks. If $\phi(\{x, y, z\})=i$, where $x<y<z$, then call $x y$ a left pair and $y z$ a right pair for color $i$. Since $\phi$ avoids $P_{4}^{3}$, no pair $x y$ is a left and right pair for the same color.

To define the $(t+2)$-track representation of $L\left(K_{n}\right)$, we use a "left" track, a "right" track, and track $i$ for $1 \leq i \leq t$. Let $\varepsilon=1 / 3$. For $x y \in E\left(K_{n}\right)$ with $x<y$, assign the interval $[x-\varepsilon, x+\varepsilon]$ in the left track and the interval $[y-\varepsilon, y+\varepsilon]$ in the right track. Assign the interval for $x y$ in track $i$ as follows:

1. If $x y$ is a left pair for $i$, then use the interval $[y-\varepsilon, y+\varepsilon]$.

2. If $x y$ is a right pair for $i$, then use the interval $[x-\varepsilon, x+\varepsilon]$.

3 . If $x y$ is not a left or right pair for $i$, then use an interval intersecting no others.

An edge in $L\left(K_{n}\right)$ involves three vertices of $K_{n}$, say $\{x, y, z\}$ with $x<$ $y<z$. The edge $\{x y, y z\}$ is represented in track $i$, where $i=\phi(\{x, y, z\})$. The edge $\{x y, x z\}$ is represented in the left track, and $\{x z, y z\}$ is represented in the right track. No non-edges (disjoint pairs $x y$ and $z w$ ) are represented.

Corollary 3.3 and the lower bound on $\mathrm{OR}_{t}\left(P_{4}^{3}\right)$ (Theorem 1.2) together yield the following:

Corollary 3.5. $\Omega\left(\frac{\lg \lg n}{\lg \lg \lg n}\right) \leq \tau\left(L\left(K_{n}\right)\right) \leq O(\lg \lg n)$.

Improving the upper bound on $\mathrm{OR}_{t}\left(P^{\prime}\right)$ would improve the lower bound on $\tau\left(L\left(K_{n}\right)\right)$, the aim being to eliminate the denominator. Both $\operatorname{OR}_{t}\left(P_{6}^{3}\right)$ and $\mathrm{OR}_{t}\left(P_{4}^{3}\right)$ are in $2^{2^{\Theta(t)}}$. Since $P^{\prime}$ propertly contains $P_{6}^{3}$, the ordered Ramsey number of $P^{\prime}$ may be larger than that of $P_{6}^{3}$. However, if it is not too much larger and also satisfies $\operatorname{OR}_{t}\left(P^{\prime}\right) \in 2^{2^{\Theta(t)}}$, then the lower bound in Corollary 3.5 improves to $\Omega(\lg \lg n)$. 


\section{Acknowledgment}

The authors thank Paul Horn and Vojtech Rödl for up-to-date information on hypergraph Ramsey numbers, Jacob Fox for pointing out the prior work on ordered Ramsey numbers, and Timothy D. LeSaulnier for contributions to early conversations establishing the unboundedness of $\tau\left(L\left(K_{n}\right)\right)$.

\section{Note added in proof}

The authors have recently learned of related earlier work. In 1992, Duffus, Lefmann, and Rödl (published as "Shift graphs and lower bounds on Ramsey numbers $r_{k}(l ; r)$ ", Discrete Math. 137 (1995), 177-187) used ordered Ramsey numbers of tight paths (in the language of "shift graphs") to obtain lower bounds on standard Ramsey numbers. In our notation, they proved $\operatorname{OR}_{t}\left(P_{r}^{k}\right) \geq$ tow $_{k-2}^{*}\left(m^{\lfloor t / 2\rfloor}+1\right)$, where $m=r-k+1$ and tow ${ }_{h}^{*}(x)$ is a variant of tow $h(x)$ in which the constant 2 is replaced by a value $c$ that is always at least $\sqrt{2}$ and approaches 2 as $t+m$ grows. Their proof relates $\operatorname{OR}_{t}\left(P_{r}^{k}\right)$ to the chromatic number of the directed graph obtained from the transitive orientation of $K_{n}$ by iterating the line digraph operator $k-2$ times. Their use of ordered Ramsey theory is mostly implicit, but in their Theorem 5 they study $\operatorname{OR}_{t}\left(P_{k+1}^{k}\right)$ explicitly. Along with tower-type lower bounds when $t \geq 3$, they also proved $\mathrm{OR}_{2}\left(P_{k+1}^{k}\right) \leq 2 k+1$. Note that for $\mathrm{OR}_{2}\left(P_{k+1}^{k}\right)$, the poset $Q_{k}$ as defined in our Theorem 2.1 is easily determined; it is obtained from a chain with $2 k-1$ elements by replacing the center element with an antichain of size 2 . Thus $\left|Q_{k}\right|=2 k$ and $\mathrm{OR}_{2}\left(P_{k+1}^{k}\right)=2 k+1$.

\section{References}

[1] M. Balko and K. Král, Ramsey numbers of ordered graphs, preprint available as arXiv:1310.7208v2.

[2] M. Baym and D. B. West, Bounds on the $k$-dimension of products of special posets, Order 30 (2013), 779-796. MR3116486

[3] S. A. Choudum and B. Ponnusamy, Ordered Ramsey numbers, Discrete Mathematics 247 (2002), 79-92. MR1893019

[4] J. Cibulka, P. Gao, M. Krčál, T. Valla, and P. Valtr, On the Geometric Ramsey number of outerplanar graphs, J. Discrete and Comp. Geometry 53 (2015), 64-79. MR3293489

[5] D. Conlon, J. Fox, and B. Sudakov, Hypergraph Ramsey numbers, J. Amer. Math. Soc. 23 (2010), 247-266. MR2552253 
[6] M. Eliáš and J. Matoušek, Higher-order Erdős-Szekeres theorems, Adv. Math. 244 (2013), 1-15. MR3077863

[7] P. Erdős and G. Szekeres, A combinatorial problem in geometry, Compositio Math. 2 (1935), 463-470. MR1556929

[8] P. Erdős and R. Rado, Combinatorial theorems on classifications of subsets of a given set, Proc. Lond. Math. Soc. 3 (1952), 417-439. MR0065615

[9] J. Fox, J. Pach, B. Sudakov, and A. Suk, Erdős-Szekeres-type theorems for monotone paths and convex bodies, Proc. London Math. Soc. 105 (2012), 953-982. MR2997043

[10] D. Gonçalves, Caterpillar arboricity of planar graphs, Discrete Math. 307 (2007), 2112-2121. MR2326172

[11] D. Gonçalves and P. Ochem, On star and caterpillar arboricity, Discrete Math. 309 (2009), 3694-3702. MR2528046

[12] A. Gyárfás and D. B. West, Multitrack interval graphs, Proc. 26th SE Intl. Conf. Comb., Graph Th., and Comput. (Boca Raton, FL, 1995) Congr. Numer. 109 (1995), 109-116. MR1369300

[13] D. Heldt, K. Knauer, and T. Ueckerdt, Track-number and caterpillar arboricity of graphs of fixed treewidth, preprint.

[14] M. Jiang, Recognizing $d$-interval and $d$-track interval graphs, Proc. 4th Intl. Conf. Frontiers in Algorithmics (Berlin, Heidelberg, FAW'10) (Springer-Verlag, 2010), 137-146. MR2684949

[15] A. D. Korshunov, The number of monotone Boolean functions, Problemy Kibernet. 38 (1981), 5-108, 272. (Russian). MR0640855

[16] D. J. Kleitman and G. Markowsky, On Dedekind's problem: the number of isotone Boolean functions, II, Trans. Amer. Math. Soc. 213 (1975), 373-390. MR0382107

[17] A. V. Kostochka and D. B. West, Every outerplanar graph is a union of two interval graphs, Proc. 30th SE Intl. Conf. Comb. Graph Th. Comput. (Boca Raton FL, 1999) Congr. Numer. 139 (1999), 5-8. MR1744225

[18] G. Moshkovitz and A. Shapira, Ramsey theory, integer partitions and a new proof of the Erdős-Szekeres theorem, preprint available as arXiv:1206.4001. 
[19] F. P. Ramsey, On a problem of formal logic, Proc. Lond. Math. Soc. 30 (1930), 264-286. MR1576401

[20] A. Seidenberg, A simple proof of a theorem of Erdős and Szekeres, $J$. London Math. Soc. 34 (1959), 352. MR0106189

Kevin G. Milans

West ViRginia University

USA

E-mail address: milans@math.wvu.edu

Derrick Stolee

Iowa State University

USA

E-mail address: dstolee@iastate.edu

Douglas B. West

ZheJiang Normal University

JiNHUA

CHINA

UNIVERSITY OF ILLINOIS

URBANA, IL

USA

E-mail address: dwest@math.uiuc.edu

URL: http://www.math.illinois.edu/ ${ }^{\text {dwest }}$

Received 12 November 2014 